\title{
Eye diseases in travelers
}

\section{Krzysztof Korzeniewski ${ }^{1,2}$ (1)}

${ }^{1}$ Department of Epidemiology and Tropical Medicine, Military Institute of Medicine, Warsaw, Poland

${ }^{2}$ Polish Society of Maritime, Tropical and Travel Medicine, Gdynia, Poland

\begin{abstract}
Travelling has been growing in popularity over the last several decades. Eye diseases, e.g. decreased visual acuity, inflammatory or degenerative lesions, chronic diseases or eye trauma, affect all groups of travelers. The main risk factors contributing to the manifestation or exacerbation of common ocular diseases include exposure to dry air (inside the airplane cabin or in air-conditioned hotel rooms), exposure to chlorinated or salty water (swimming/bathing in swimming pools or in the sea), and sudden changes in the weather conditions. In addition, travelers to tropical destinations are at risk of ocular diseases which are rarely seen in temperate climate, e.g. onchocerciasis, loiasis, gnatostomosis, African trypanosomosis, or trachoma. The most common condition of the eye seen in travelers is conjunctivitis; it may be either of cosmopolitan (bacterial or viral infections, allergic inflammation) or tropical etiology, e.g. arboviral infections (zika, chikungunya). Given the fact that a large proportion of the general population have decreased visual acuity and many of them wear contact lenses rather than glasses, keratitis has become a common health problem among travelers as well; the major risk factors in such cases include sleeping in contact lenses, prolonged exposure to air-conditioning, working with a computer or swimming/bathing in microbiologically contaminated water (e.g. Acanthoamoeba protozoa). Conditions affecting the cornea, conjunctiva or lens may also occur due to excessive exposure to solar radiation, especially if travelers wear glasses without a UV protection.
\end{abstract}

(Int Marit Health 2020; 71, 1: 78-84)

Key words: eye diseases, travelers, treatment, prevention

\section{EPIDEMIOLOGY}

The World Health Organization (WHO) has estimated that over 280 million people globally are affected by visual defect, of whom $14 \%$ have blindness and another $86 \%$ have less severe ocular problems, e.g. decreased visual acuity or inflammatory conditions. The leading causes of sight defect globally include refractive error and cataract (clouding of the lens). The highest prevalence of eye conditions is reported from China and India, i.e. the two most populous countries in the world. Population ageing is considered the major risk factor for increasing prevalence of visual defect. More than $80 \%$ of the general population over the age of 60 years old has vision defect, usually presenting as decreased acuity (presbyopia). According to the data from the statistics in Poland as much as $50 \%$ of adults declare that they wear glasses or contact lenses to correct distance or near vision defect (in the age group 50-59 it is $76 \%$, and in the age group > 60 more than $80 \%$ ). Among children under 15 years old, visual defect is seen in $50 \%$ of boys and more than $60 \%$ of girls. In Poland, the most common diseases of the eye include cataract, glaucoma, age-related macular degeneration (AMD) and diabetic retinopathy. More than 800,000 Poles (mostly patients over the age of 60 ) have cataract and this number is likely to grow. In 2020 , it is expected to exceed one million cases in 38.5 million population. Clouding of the lens is more often seen in women ( $70 \%$ cases) than in men. Another common eye condition, i.e. glaucoma affects over 450,000 Poles, mostly women (70\% cases), and the number of cases is constantly increasing. It is expected to reach over 600,000 cases in 2035 . The data from the statistics in Poland also shows there are 1.9 million diagnosed cases of AMD, including 130,000 cases of the more advanced form of the disease, i.e. wet AMD potentially leading to vision loss (the risk increasing with age). The major risk factors for AMD

Prof. Krzysztof Korzeniewski, MD, PhD, Chairman of the Polish Society of Maritime, Tropical and Travel Medicine, Powstania Styczniowego St. 98, 81-519 Gdynia, Poland; Military Institute of Medicine, Head of the Department of Epidemiology and Tropical Medicine, Szaserów St. 128, 04-141 Warszawa, Poland, 
include smoking, arterial hypertension, high cholesterol concentration and obesity. Diabetes mellitus (DM) is one of the most common chronic diseases in Poland affecting over 2 million people. Chronic hyperglycemia causes damage to the retina and thus plays a major role in the development of diabetic retinopathy. The disease affects approximately $90 \%$ of patients with type $1 \mathrm{DM}$ and $60 \%$ of patients with type $2 \mathrm{DM}$ who have had the disease for 20 years (diabetic retinopathy is the major cause of vision loss in patients over 65 years old), it also leads to diabetic macular edema. The data from the statistics indicates that there are 4.9 million disabled people living in Poland of whom $35 \%$ are affected by an ocular disease or have suffered an eye trauma (over 60,000 of Poles over the age of 15 years old have blindness or severe visual impairment). In general, the diagnosis of ocular conditions may be problematic because a large number of diseases affecting the eye tend to be asymptomatic at an early stage. In addition, screening for visual defects is rare in Poland (around $30 \%$ of Polish people have never been consulted by an ophthalmologist). With such a high number of patients with visual defects in the general population, it is not surprising that conditions of the eye are quite common among travelers as well [1-6].

\section{CLINICAL PICTURE}

The International Classification of Diseases ICD-10 classifies the diseases of the eye and adnexa into 11 categories: disorders of eyelid, lacrimal system and orbit; disorders of conjunctiva; disorders of sclera, cornea, iris and ciliary body; disorders of lens, disorders of choroid and retina; glaucoma; disorders of vitreous body and globe; disorders of optic nerve and visual pathways; disorders of ocular muscles, binocular movement, accommodation and refraction; visual disturbances and blindness; other disorders of the eye and adnexa. Eye conditions may also arise from injuries, poisoning or congenital disorders; however, those cases are classified outside the category listing the diseases of the eye and adnexa [1].

A great majority of patients with eye problems are treated on an out-patient basis and the most prevalent conditions include inflammation of the protective apparatus of the eye or the globe which often manifest with pain, redness, swelling of the eyelid or the globe or discharge from the conjunctival sac. In such cases patients usually complain of foreign body sensation, tearing or itching. When visual acuity is not decreased, pupils react normally and the cornea is transparent, the symptoms are suggestive of conjunctivitis or inflammation of the eyelid. If, however, the symptoms occur seasonally, it may be suggestive of an allergic reaction. In such cases it is recommended to use antihistamines rather than antibiotic or corticosteroid drops/ointments. Although they may initially reduce the symptoms, they are likely to have adverse effects on the eye in the longer perspective (dysfunction of the tear film or dysfunction of the secretory glands in the eyelid or the conjunctiva) [5].

\section{DRY EYE SYNDROME}

It is one of the most common conditions affecting the eye. It usually occurs due to inappropriate or prolonged use of topical medications for the treatment of conjunctivitis or blepharitis (inflammation of the eyelid), insufficient tear production or alternations in the quality or quantity of the tear film. The condition is associated with inadequate wetting of the cornea and the conjunctiva which causes discomfort (dry eye sensation, burning, foreign body sensation) but occasionally may lead to more severe conditions of the globe. Dry eye syndrome is a disease reflecting the modern way of life where much time is spent in poorly-ventilated or air-conditioned rooms and in front of a computer screen. Under such conditions lacrimal glands do not produce sufficient tears to compensate for the excessive tear evaporation. The major risk factors contributing to the occurrence of the dry eye syndrome during travel include: prolonged exposure to dry air (in an airplane), difficult weather conditions (low humidity, dry wind, dust) exposure to salty or chlorinated water (swimming/bathing in a swimming pool or in the sea) [5, 7-9].

\section{EXCESSIVE TEAR PRODUCTION (WATERY EYES)}

The condition (often unilateral) may be suggestive of a blocked tear duct. Acute pain, swelling and redness in the corner of the lower eyelid may be indicative of dacryocystitis (inflammation of the tear sac). Treatment of a tear drainage blockage usually requires ophthalmological/surgical intervention $[5,10]$.

\section{INFLAMMATION OF THE EYE}

Inflammatory lesions affecting the eye are the most common ocular conditions. They typically arise from the dysfunction of the glands which are part of the protective apparatus of the eye (the sebaceous glands of Zeis and the sweat glands of Moll in the eyelash follicles, Meibomian glands in the tarsal plate of the upper eyelid), the conjunctiva (lining the inside of the eyelid and the sclera) or the lacrimal apparatus. The infection often occurs as a result of a gland blockage (hordeolum, dacryocystitis, abscess of the eyelid) or a mechanical injury e.g. contact lens associated damage to the corneal epithelium (increased susceptibility to infections). Some inflammatory conditions may be of endogenous nature (when an infection which has already affected other organs or systems spreads to the eye). An eye infection may subsequently be carried via the angular vein and the cavernous sinus to the meninges causing neurological disorders [11]. 
Conjunctivitis. The condition usually affects both eyes and is the effect of either external (bacteria, viruses, allergens) or internal factors. The common signs and symptoms include burning sensation, foreign body sensation, stinging, photophobia, tearing, redness and purulent, mucous or watery discharge. The condition may be mild to severe with symptoms ranging from slight redness to massive discharge. Conjunctivitis may be self-limiting and even if it is left untreated the signs and symptoms usually subside within 2 weeks of onset. Nevertheless, patients will benefit from an early diagnosis and prompt treatment as it may help prevent chronic conjunctivitis or keratitis in the future [11].

Keratitis. The condition usually presents with severe clinical signs and symptoms (decreased visual acuity, pain, photophobia, tearing, discharge, visible corneal infiltrate). Keratitis may be of infectious or non-infectious etiology and it may lead to loss of vision. In temperate climate zones keratitis is typically of bacterial etiology, while in the tropical climate - it is usually caused by a fungal or a mixed infection. In patients wearing contact lenses inflammatory lesions (resulting from damage to the corneal surface) are usually associated with Pseudomonas aeruginosa infection. The common risk factors for keratitis include bacterial or viral infections of the eye surface, injuries, diabetes, and immunosuppressive treatment. Inflammation manifesting with tissue loss and corneal infiltrates may lead to scarring and perforation of the cornea and eventually to loss of vision [11].

Orbital cellulitis. It is commonly caused by the spread of an infection from the paranasal sinuses but it may also occur as a complication after a sinus or ocular surgery. The condition is serious (the risk of a generalized infection) and requires treatment on an in-patient basis [12].

Hordeolum. It is an infection of the eyelid glands (glands of Zeis, Moll or meibomian glands), which is usually caused by Staphylococcus aureus. Hordeolum may be either external or internal; the former results from obstruction and infection of an eyelash follicle and adjacent glands of Zeis or glands of Moll, while an internal hordeolum, results from an infection of a meibomian gland (tarsal glands), which is responsible for the production of the tear film lipid layer [12].

Chalazion. It is a chronic infection of a meibomian gland caused by obstruction of the gland and accumulation of lipid material in the eyelid leading to the formation of a cyst usually associated with redness and swelling. If a chalazion persists for more than several weeks despite treatment (topical antibiotic and hot compresses), the recommended treatment of choice remains incision and curettage [12].

Blepharitis. It is a bilateral chronic infection of the eyelid causing irritation of the eye and burning sensation. It is often associated with conjunctivitis, keratitis, formation of intrafollicular abscesses at the base of the eyelashes and disruption of tear secretion. Chronic blepharitis may lead to the growth disorder, loss or discoloration of the eyelashes [12].

Foreign body in the eye. It is usually a grain of sand, a speck of dust or a very small insect (e.g. sand fly), its presence causes discomfort, tearing, redness and pain. If patients are unable to remove the foreign body from the eye on their own, they are recommended to seek medical assistance [5].

On top of the conditions discussed above, sudden vision impairment may be associated with some systemic diseases e.g. diabetes, trauma or thrombotic lesions.

\section{DIABETIC RETINOPATHY}

It may happen that an ophthalmologist will be the first physician to diagnose a patient with diabetes (examination of the fundus). This may be the case when a patient reports to a specialist due to sudden vision impairment, which may potentially be associated with vitreous or retinal hemorrhage. Diabetic macular edema may also be responsible for sudden vision impairment in diabetic patients; mild diabetic macular edema may be associated with slightly decreased visual acuity, whereas its severe form may lead to vision loss [4].

\section{RETINAL VASCULAR OCCLUSIONS}

The condition usually occurs in the elderly patients with a history of atherosclerosis, diabetes, or arterial hypertension but may also be found in women at the childbearing age using hormonal contraceptives. The central retinal vein occlusion (CRVO) results in sudden vision impairment and requires immediate hospital treatment, whereas a branch retinal vein occlusion (BRVO) is a mild condition which may give no symptoms and may go unnoticed. In most cases both the CRVO and BRVO are unilateral $[4,13]$.

\section{RETINAL HEMORRHAGE}

It is a disorder of the retina which commonly occurs at high altitudes, e.g. during high altitude climbing. Exposure to hypobaric hypoxia increases retinal blood flow and leads to retinal hemorrhages (the retina is highly sensitive to hypoxic conditions). The condition is often asymptomatic, but may sometimes be associated with visual disturbances (seeing floaters or flashes of light, blurred vision, decreased acuity) due to bleeding at or around the macula. Retinal hemorrhage was found to occur in approximately $30 \%$ of climbers who reach the altitude of $4200 \mathrm{~m}$ a.s.l., $50-60 \%$ of climbers at $5500 \mathrm{~m}$ a.s.l., and supposedly in $100 \%$ climbers at 6800 m. a.s.l. If retinal hemorrhage occurs, the ophthalmologic examination will reveal optic disc hyperemia and increased vessel tortuosity in the fundus of the eye. In most cases the condition is self-limiting and retinal bleeding usually stops after 1-2 weeks [14]. 


\section{RETINAL DETACHMENT}

The disorder is clinically silent and often unilateral. It leads to sudden visual impairment; it usually affects the elderly patients with advanced myopia. In younger patients retinal detachment is most commonly trauma-related (the eye or head injury). Patients with retinal detachment report a decrease in the field of vision. If the macula is not involved, central vision may be preserved; however, if the macula is involved, the prognosis is much worse and lesions may be irreversible. Retinal detachment is an emergency condition and requires immediate surgical intervention [4].

\section{EYE TRAUMA}

An injury to the eye may be the result of a traffic accident as well as a sports injury or even UV radiation. It is strongly recommended that all travelers, both children and adults wear certified sunglasses for UV protection [15].

\section{DISEASES OF THE EYE IN HOT CLIMATE CONDITIONS}

Travelers to tropical or subtropical destinations, with high prevalence of pathogens or infection vectors which are specific for the hot climate areas, may develop diseases of the eye which are rarely seen in temperate climate conditions. Such conditions include visual impairment characteristic for onchocerciasis (Onchocerca volvulus microfilariae migrate from the skin into the conjunctiva, cornea and anterior chamber of the eye leading to keratitis, anterior and intermediate uveitis (inflammation of the iris and the ciliary body) and eventually to vision loss); conjunctivitis/foreign body in the eye in loiasis (Loa loa microfilariae penetrate into the subconjuntival tissues), swelling of the eyelid and conjunctiva in gnatostomosis, swelling of the eyelids in African trypanosomosis, or conjunctivitis and keratitis in trachoma. Of all the eye conditions seen in travelers to tropical or subtropical destinations conjunctivitis is the most common; in such cases conjunctivitis is either of cosmopolitan (bacterial or viral infections, allergic inflammation) or tropical e.g. arboviral infections (zika, chikungunya) etiology [16-21].

\section{TREATMENT}

The treatment of any diseases of the eye (e.g. decreased visual acuity, acute or chronic inflammatory conditions, degenerative lesions or eye trauma) requires consultation with an ophthalmologist. This must be remembered by all travelers but especially those travelling to countries where specialist healthcare is unavailable or difficult to access (a professionally equipped office of an ophthalmologist vs. a primary health care provider in remote areas of Asia or Africa). Therefore, it is recommended that travelers with underlying eye conditions take good preparations before going on a journey. They must not forget to bring their glass- es or contact lenses, all the regularly taken and emergency medications as well as products for eye care (drops and wipes). If, nevertheless, eye or vision problems do occur during travel, the traveler will have to assess the nature of the condition and its severity. If the lesions are associated with exposure to external or environmental factors and the condition is mild enough to be managed individually with the available medications, there is no need to seek medical help. If, however, the lesions are more severe, travelers are strongly recommended to report to a medical facility for a consultation with an ophthalmologist (eye examination, specialist treatment). In order to establish an accurate diagnosis, an ophthalmologist must have access to the specialist ophthalmic diagnostic equipment (an ophthalmoscope for the fundus examination, a slit lamp to inspect the anterior segment of the eye/eyelids, conjunctiva, sclera, iris, lens, cornea/, tonometer to determine the intraocular pressure, etc.) [22]. The type of treatment will depend on the condition itself, the outcome of the clinical examination as well as the results of diagnostic tests. Intraocular and orbital infections of bacterial etiology require the use of systemic antibiotic therapy and the parenteral administration of antimicrobial agents. Post-traumatic or post-surgical anterior uveitis is managed with subconjunctival injections (the drug administered in this way reaches the iris, the ciliary body and the cornea through the sclera route). In case of an intraocular inflammation, the drug is injected into the anterior chamber or the vitreous humor. Topical medications (e.g. drops, suspensions and ointments) are used for the treatment of infections/inflammation of the anterior segment of the eye (strict compliance with hygiene recommendations is essential in such cases). The drugs administered into the conjunctival sac are absorbed through the cornea (topical medications may enter into the systemic circulation via the highly vascularized conjunctiva and further via the lacrimal sac and may potentially cause adverse reactions; therefore, patients must strictly comply with the recommended dosage regimens and respect the expiry date and the period after opening) [8, 12, 22, 23].

\section{PREVENTION}

In general, travelers are likely to be exposed to a number of risk factors which may result in the manifestation of acute eye conditions or the exacerbation of underlying ophthalmological diseases. The most common risk factors which cause irritation (dry eyes, burning sensation, redness) of the eye or its protective apparatus include prolonged exposure to air-conditioning (inside an airplane cabin or in a hotel room), wind, low humidity, chlorinated water in the swimming pool or salty water in the sea. If the eyes feel dry or irritated, travelers may use re-wetting eye drops. Artificial tears may be used both for prevention and treatment of 
the dry eyes symptoms. A good way to stabilize the lipid layer of the tear film and prevent its evaporation is to use eye drops containing sodium hyaluronate. The drops lubricate the corneal surface and may be used with all types of contact lenses. Moisturizing eye drops are also recommended to patients with certain chronic conditions such as Sjögren's syndrome, hormonal imbalance (menopause) or patients with a history of an ophthalmologic surgery [24, 25]. Travelers who are likely to be exposed to difficult climate conditions (high temperatures, humidity, air pollution) are recommended to take good care not only of the eyes but also of their protective apparatus. A good solution is to use sterile eyelid wipes (e.g. Blephaclean) for everyday hygiene of the eyelashes and eyelids. The wipes show antibacterial, anti-inflammatory and regenerative effect and can also be used for make-up removal [22].

\section{DRY EYE SYNDROME}

Artificial tear drops are the first line treatment for patients with dry eye symptoms (the medication is short-acting and should be applied at regular intervals, several times a day; artificial tear drops should be free from preservatives as they may irritate the eye). Apart from using the right medications, one should also remember about some simple preventive measures to reduce tear film evaporation (wearing large frame glasses which provide better protection from the wind, dust or the sun; placing the computer screen below the eye level to narrow the eye opening; trying to reduce smoking and avoiding smoke-filled rooms, using rewetting eye drops while staying or working in air-conditioned rooms) [26-28].

\section{SUNGLASSES}

Sunglasses are an essential travel item, especially when travelling to areas where UV exposure is high. The most important sunglasses component is obviously the UV filter. When a person wears darkened glasses, pupils dilate because less light reaches the eye; therefore, wearing sunglasses without a UV filter actually increases the exposure to UV radiation as more sunlight enters the eye through dilated pupils. Excessive UV radiation may cause damage to the cornea, lens or retina. Travelers with a vision defect are recommended to wear both their contact lenses and sunglasses with a UV filter. If, for some reason, travelers cannot wear contact lenses (inflammatory lesions, swimming/bathing in the water) they are advised to purchase darkened prescription glasses or glasses with photochromic lenses (darkening when exposed to the sun). Sunglasses which provide protection against the UV radiation should be labeled (on the lens or frames) as 'UV400', '100\% UV reduction', '100\% block' or 'UV 100\%'. In line with current recommendations, the UV protection filters fall into four distinct categories, depending on the level of UV transmis- sion. Category 1 (43-80\% transmission) or Category 2 are recommended on cloudy days (sun radiation is lower but still present), Category 3 (typical for most types of sunglasses) is recommended on sunny days, this category is also recommended to drivers (drivers are suggested to wear sunglasses with polarized lenses which additionally eliminate the glare). Category 4 sunglasses (3-8\% transmission) are recommended to be worn in intense sunshine e.g. in snow covered mountains; they are not to be used while driving as the lens are too dark. Travelers need to keep in mind that sunglasses which are sold in the developing countries may be of low quality. Therefore, before buying a pair of sunglasses abroad it is important to check whether the lens have been certified (those which meet the requirements will be marked as follows: EN 166:2001, PN-EN ISO 123121:2014-02, 89/686/EWG, ISO 9001:2000) [22, 29-32].

\section{TRAVELLING WITH CONTACT LENSES}

Given the fact that a considerable proportion of travelers have decreased visual acuity and many are contact-lens wearers, it seems reasonable to discuss some general guidelines for travelling with contact lenses. First, contact lens wearers are strongly recommended to bring not only their contact lenses but also a pair of glasses as a back-up. Wearing glasses may be more comfortable then wearing contacts in some situations, e.g. during a long flight lasting 6-8 hours or more. Low humidity inside an airplane is primarily the effect of the air conditioning but also of high altitude conditions (the cabin pressure corresponds to an altitude of 1800-2400 m a.s.l.). What is more, sleeping on the plane will be much more comfortable with lenses removed. Another common problem for contact lens wearers is the exposure to dry air (e.g. an air conditioned hotel room). Dry air may cause discomfort and irritation so it is very important to keep your eyes moisturized by using rewetting drops/ /artificial tear drops or by wearing moisturized lenses. When travelling to sunny destinations (the seaside beach, high mountains, a skiing holiday), contact lens wearers must not forget about adequate UV protection. Some manufacturers are now marketing contact lenses which block the UV radiation and thus provide protection against the harmful effects of the sun [33]. Also, travelers who wear contact lenses need to take certain precautions when swimming in the pool or in the sea. The best solution is to avoid wearing contact lenses in the water. This is because contact lenses increase the risk of infection with the pathogenic microorganisms which may be present in the water. Although generally not harmful to the skin, the respiratory or the gastrointestinal system, some pathogens which are found in the water (Acanthoamoeba protozoa) may contaminate the lens and consequently lead to corneal inflammation [34]. Travelers must also remember to follow the contact lens hygiene recommendations (this 
particularly applies to extended wear contacts), i.e. never use tap water to clean the lenses or the lens storage case (these may only be cleaned with contact lens solution) and replace the storage case at intervals recommended by the manufacturer. Compliance with the storage recommendations and replacement schedules is particularly important in tropical and subtropical destinations, where microbial contamination of water is common. In order to minimize the risk of the eye infection, travelers are recommended to switch to daily disposable lenses for the duration of their trip. Obviously, travelers will not feel comfortable wearing contact lenses if they do not follow the very basic hygiene practices such as washing their hands with soap and water or using hand sanitizer before handling the lenses or putting them in. If a traveler opts for extended wear contact lenses, they need to bring at least one extra pair in case their contacts get lost or damaged (the same applies to glasses wearers; people with vision defect should never travel without an extra pair of contacts or eyeglasses). Travelers are also reminded to keep their contacts/glasses, contact solution or eye-drops in their carry-on baggage so as not to lose them. When travelling by plane it should be reminded that all liquids must be stored in individual containers of no more than $100 \mathrm{~mL}$ and packed in a transparent plastic bag with a seal (e.g. a zip-lock) [22].

\section{REFERENCES}

1. Raciborski F, Kłak A, Gawińska E, et al. Eye diseases - health, social and civilization challenge in the face of population aging [in Polish]. The Healthcare Institute, Warszawa 2016.

2. Statistics Poland. Health condition of the Polish population in 2014. GUS Warszawa, 2016 [in Polish]. http://stat.gov.pl/obszary-tematyczne/zdrowie/zdrowie/stan-zdrowia-ludnosci-polski-w-2014-r-,6,6. html.

3. Partyka O, Wysocki MJ. Epidemiology of eye diseases and infrastructure of ophthalmology in Poland. Przegl Epidemiol. 2015; 69(4): 905-908, indexed in Pubmed: 27139360.

4. Omulecki W, Romaniuk W, Samoliński B, et al. Ophthalmology and retinal diseases in the aspect of healthy and active aging [in Polish]. Foundation for Healthy Aging. Warszawa 2015.

5. Stankiewicz A. Most important (most common) ophthalmic disorders in family doctor practice [in Polish]. Fam Med Primary Care Rev. 2013; 15(3): 477-482.

6. Szmurło D, Fundament T, Kopeć G, et al. Access to ophthalmologic care in Poland. A systematic approach [in Polish]. Central and Eastern European Society of Technology Assessment in Health Care. Kraków 2013.

7. Ezuddin NS, Alawa KA, Galor A. Therapeutic Strategies to Treat Dry Eye in an Aging Population. Drugs Aging. 2015; 32(7): 505-513, doi: 10.1007/s40266-015-0277-6, indexed in Pubmed: 26123947.

8. Gadaria-Rathod N, Lee KI, Asbell PA. Emerging drugs for the treatment of dry eye disease. Expert Opin Emerg Drugs. 2013; 18(2): 121-136, doi: 10.1517/14728214.2013.791676, indexed in Pubmed: 23621500.

9. Hessen M, Akpek EK. Dry eye: an inflammatory ocular disease. J Ophthalmic Vis Res. 2014; 9(2): 240-250, indexed in Pubmed: 25279127.
10. Zhou L, Beuerman RW. Tear analysis in ocular surface diseases. Prog Retin Eye Res. 2012; 31(6): 527-550, doi: 10.1016/j.preteyeres.2012.06.002, indexed in Pubmed: 22732126.

11. Kański JJ, Pavesio CE, Tuft SJ. Ed.). Inflammatory eye diseases [in Polish]. Elsevier Urban \& Prater, Wrocław 2008.

12. Antoniak K. Pharmacotherapy of bacterial infections of the eye [in Polish]. Advances in Pharmacotherapy. 2009; 65(2): 124-131.

13. Klein R, Klein BEK. The prevalence of age-related eye diseases and visual impairment in aging: current estimates. Invest Ophthalmol Vis Sci. 2013; 54(14): ORSF5-ORSFORSF13, doi: 10.1167/iovs.1312789, indexed in Pubmed: 24335069.

14. Korzeniewski K. [Health problems in high mountain conditions]. Pol Merkur Lekarski. 2008; 25(146): 161-165, indexed in Pubmed: 18942339.

15. Wang F, Hu L, Gao Q, et al. Risk of ocular exposure to biologically effective UV radiation in different geographical directions. Photochem Photobiol. 2014; 90(5): 1174-1183, doi: 10.1111/php.12287, indexed in Pubmed: 24804634.

16. Khairallah M, Kahloun R. Ocular manifestations of emerging infectious diseases. Curr Opin Ophthalmol. 2013; 24(6): 574-580, doi: 10.1097/ICU.0b013e3283654e09, indexed in Pubmed: 24030241.

17. Das D, Ramachandra V, Islam S, et al. Update on pathology of ocular parasitic disease. Indian J Ophthalmol. 2016; 64(11): 794-802, doi: 10.4103/0301-4738.195590, indexed in Pubmed: 27958200.

18. Diaz JH. Ocular Filariasis in US Residents, Returning Travelers, and Expatriates. J La State Med Soc. 2015; 167(4): 172-176, indexed in Pubmed: 27159510.

19. Pleyer U, Klauß V, Wilking H, et al. [Tropical ophthalmology: Intraocular inflammation caused by "new" infectious pathogens and travel-related infections]. Ophthalmologe. 2016; 113(1): 35-46, doi: 10.1007/s00347-015-0176-6, indexed in Pubmed: 26649438.

20. Ryan ET, Durand M. Ocular Disease. In: Guerrant R.L., Walker D.H., Weller P.F. Tropical Infectious Diseases (Eds). 2nd Edition. Saunders Elsevier 2006: 1554-1600.

21. Yorston DH, McGavin DD. Ophthalmology in the Tropics and Subtropics. In: Cook G.C., Zumla A. (Eds). Manson's Tropical Diseases. 22nd Edition. Saunders Elsevier 2009: 283-332.

22. Korzeniewski K. Travel medicine in medical practice [in Polish]. Travel Medicine, Gdynia 2019: 111-121.

23. Marshall LL, Roach JM. Treatment of dry eye disease. Consult Pharm. 2016; 31(2): 96-106, doi: 10.4140/TCP.n.2016.96, indexed in Pubmed: 26842687.

24. Moshirfar M, Pierson K, Hanamaikai K, et al. Artificial tears potpourri: a literature review. Clin Ophthalmol. 2014; 8: 1419-1433, doi: 10.2147/OPTH.S65263, indexed in Pubmed: 25114502.

25. Torkildsen G, Brujic M, Cooper MS, et al. Evaluation of a new artificial tear formulation for the management of tear film stability and visual function in patients with dry eye. Clin Ophthalmol. 2017; 11: 1883-1889, doi: 10.2147/OPTH.S144369, indexed in Pubmed: 29089744.

26. Leggat PA, Speare R, Moon ME. Sore eyes and travelers. J Travel Med. 1999; 6(1): 45-47, doi: 10.1111/j.1708-8305.1999. tb00866.x, indexed in Pubmed: 10071372.

27. Leibowitz HM. The red eye. N Engl J Med. 2000; 343: 345-351, doi: 10.1056/NEJM200008033430507, indexed in Pubmed: 10922425.

28. Messmer EM. The pathophysiology, diagnosis, and treatment of dry eye disease. Dtsch Arztebl Int. 2015; 112(5): 71-81; quiz 82, doi: 10.3238/arztebl.2015.0071, indexed in Pubmed: 25686388. 
29. Diaz JH, Nesbitt LT. Sun exposure behavior and protection: recommendations for travelers. J Travel Med. 2013; 20(2): 108-118, doi: 10.1111/j.1708-8305.2012.00667.x, indexed in Pubmed: 23464719.

30. Izadi M, Jonaidi-Jafari N, Pourazizi M, et al. Photokeratitis induced by ultraviolet radiation in travelers: A major health problem. J Postgrad Med. 2018; 64(1): 40-46, doi: 10.4103/jpgm.JPGM_52_17, indexed in Pubmed: 29067921.

31. Sasaki H, Sakamoto Y, Schnider C, et al. UV-B exposure to the eye depending on solar altitude. Eye Contact Lens. 2011; 37(4): 191-195, doi: 10.1097/ICL.0b013e31821fbf29, indexed in Pubmed: 21670696.
32. Tuchinda C, Srivannaboon S, Lim HW. Photoprotection by window glass, automobile glass, and sunglasses. J Am Acad Dermatol. 2006; 54(5): 845-854, doi: 10.1016/j.jaad.2005.11.1082, indexed in Pubmed: 16635665.

33. Walsh JE, Bergmanson JPG. Does the eye benefit from wearing ultraviolet-blocking contact lenses? Eye Contact Lens. 2011; 37(4): 267-272, doi: 10.1097/ICL.0b013e3182235777, indexed in Pubmed: 21670694.

34. Izadi M, Pourazizi M, Babaei M, et al. Ocular parasitosis caused by protozoan infection during travel: focus on prevention and treatment. Int J Prev Med. 2018; 9: 79, doi: 10.4103/ijpvm.IJPVM_161_18, indexed in Pubmed: 30283611. 